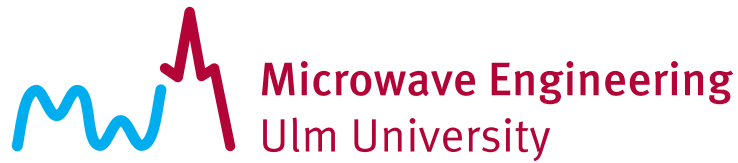

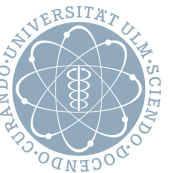

\section{Data Rate Reduction for Chirp-Sequence based Automotive Radars using Compressed Sensing}

Fabian Roos, Philipp Hügler, Christina Knill

Nils Appenrodt, Jürgen Dickmann, and Christian Waldschmidt

(C) 2018 IEEE. Personal use of this material is permitted. Permission from IEEE must be obtained for all other uses, in any current or future media, including reprinting/republishing this material for advertising or promotional purposes, creating new collective works, for resale or redistribution to servers or lists, or reuse of any copyrighted component of this work in other works. 


\title{
Data Rate Reduction for Chirp-Sequence based Automotive Radars using Compressed Sensing
}

\author{
Fabian Roos*, Philipp Hügler*, Christina Knill*, \\ Nils Appenrodt ${ }^{\dagger}$, Jürgen Dickmann ${ }^{\dagger}$, and Christian Waldschmidt* \\ ${ }^{*}$ Institute of Microwave Engineering, Ulm University, 89081 Ulm, Germany \\ ${ }^{\dagger}$ Daimler AG, Group Research and Advanced Engineering, 89081 Ulm, Germany \\ Email: fabian.roos@uni-ulm.de
}

\begin{abstract}
For autonomous driving high-resolution radar sensors are key components, which have the drawback of high data rates. In order to reduce the amount of sampled data, random samples can be omitted and afterwards reconstructed using compressed sensing methods. A possible application is that not every receiving antenna element demands its own analog-todigital converter. One converter can be used for several receiving elements with a random assignment to each antenna instead. In this paper, an analysis is presented of how many samples can be neglected such that a successful reconstruction in post-processing for an automotive scenario is possible. A measurement result is shown to prove that with only $40 \%$ of samples a successful reconstruction is possible.
\end{abstract}

\section{INTRODUCTION}

To enable fully autonomous driving, high-resolution radar sensors are required [1]. For a linear frequency modulated continuous wave modulation, also called chirp-sequence, this means a bandwidth larger than $1 \mathrm{GHz}$ [2], and several consecutive frequency ramps are evaluated by different receiving antenna elements. Usually, each receiving antenna element has its own analog-to-digital converter $(A D C)$ as shown in Fig. 1 (a). To reduce hardware effort one $A D C$ could be used for several receiving elements as shown in (b). Either whole frequency ramps from one antenna are sampled before the next antenna is selected or the switching between different antennae can be realised after a defined or random number of samples.

The application of compressed sensing allows to omit random samples and restore them afterwards if certain conditions are fulfilled [3], [4], e.g., for the radar application, the signal is sparse in the frequency domain. Additionally, in the automotive environment extended targets are present which often results in a bunch of frequencies in close range. The
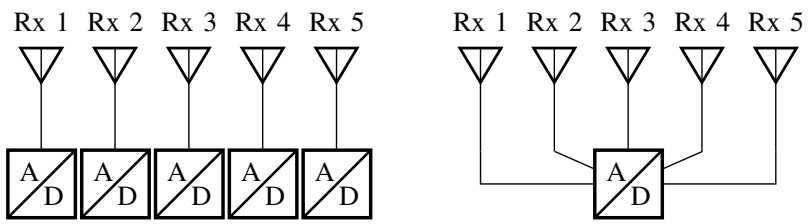

(a) Each receiving element has its own (b) Only one $A D C$ is used with a $A D C$. switching scheme.

Fig. 1. Five different receiving antenna elements are present, which are evaluated in parallel (a) with five analog-to-digital converters or using only one converter as in (b).

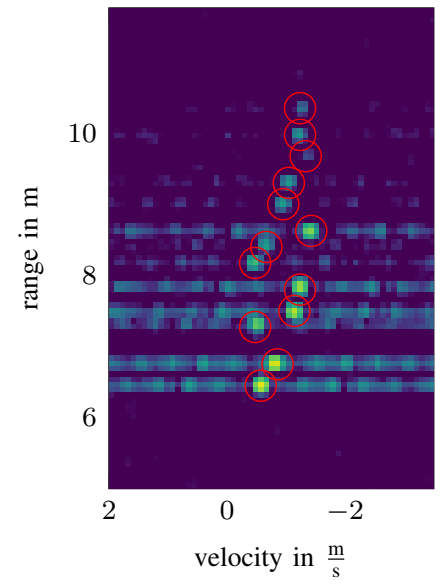

(a) Removal of whole chirps.

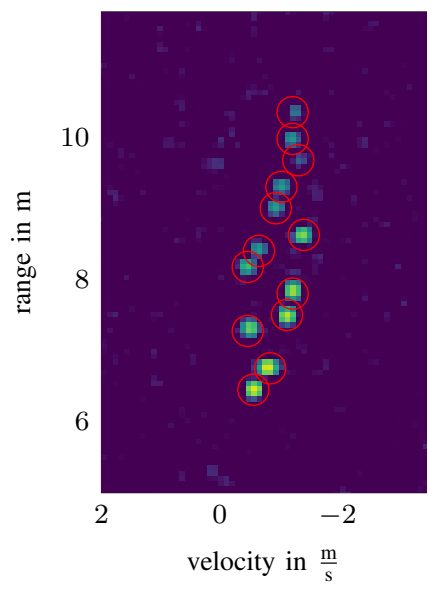

(b) Removal of random samples.
Fig. 2. To reduce the data rate, $50 \%$ of the acquired samples are removed In (a) whole chirps are removed and in (b) random samples. This results in different artefact levels for the simulated targets $(\bigcirc)$.

reconstruction algorithm should cope with these scenarios as well.

The authors of [5] are removing random chirps to reduce the amount of data resulting in higher artefact levels induced by the missing data.

In comparison to remove complete chirps, the removal of random samples has a lower influence on the rising artefact level, which originates from missing data. In Fig. 2, a rangeDoppler evaluation is shown with $50 \%$ of the data randomly removed. In (a) complete chirps are removed resulting in a rising artefact level in the same range cell, whereas the removal of random samples in (b) just increases the total noise level. This behaviour is explained for the assignment of subcarriers in $O F D M$ radar in [6]. Hence, in this paper it is proposed to remove random samples of each consecutive chirp. An analysis is presented on the influence of data reduction to the detection rate, false alarm rate, and peak power reconstruction.

\section{Signal PROCESSING}

The signal processing consists of several steps. First, the signal generation and removal of samples, second, the reconstruction of missing data, and finally, the analysis consisting of target detection and evaluation. 


\section{A. Signal Generation and Data Removal}

The chirp-sequence modulation [7] using $L$ frequency ramps with a bandwidth $B$, duration $T_{\mathrm{c}}$, and chirp repetition time $T_{\mathrm{r}}$ is applied. For the following analysis either whole random chirps or random samples are removed. The positions of the randomly removed samples are different for each ramp which prohibits a certain pattern.

\section{B. Signal Reconstruction}

To reconstruct the missing samples the iterative method with adaptive threshold (IMAT) [8] is applied. In the first step, the missing samples are assumed to be zero and then, a twodimensional fast Fourier transform $(2 D-F F T)$ is applied. This leads to a range-Doppler spectrum as in Fig. 2. As a starting threshold the maximum peak power $\beta$ is used. Every spectral peak above this threshold is used to estimate the missing time domain samples as a first approximation. In the following iterations the threshold

$$
\operatorname{thr}_{\mathrm{n}}=\beta \mathrm{e}^{-\alpha n}
$$

is exponentially decreased with $n \in[0, N-1]$ being the current iteration. As in each consecutive iteration the threshold is lowered, more frequency components are utilised to reconstruct the missing data. The parameter $\alpha$ defines how fast the threshold is reduced. It must be assured that in neither iteration the artefact induced spectral components are above the threshold, as in this case ghost targets are created. Therefore, the number of iterations is limited, such that the threshold is not lower than $10 \mathrm{~dB}$ above the noise floor to prevent an amplification of random noise. In each iteration every sample in spectrum that is classified as noise by the peak detection algorithm is used to estimate the current noise power.

\section{Signal Evaluation}

The reconstructed time domain data is used to calculate the range-Doppler spectrum. For target detection the ordered statistic-constant false alarm rate (OS-CFAR) algorithm [9] is used in combination with a peak search algorithm. In the simulation the extracted targets are matched with the known simulated ones. Hence, a detection rate is calculated as well as a false alarm rate.

\section{Simulations}

The parameters of the chirp-sequence modulation used for the simulation are given in Tab. I. For the influence analysis of missing samples and their recoverability, an extended target scenario is considered. Therefore, a vehicle is simulated using the model proposed in [10]. In contrast to classic compressed sensing applications, extended targets appear as broad peaks in the spectrum consisting of several close frequencies. A realisation with several close targets in range and velocity is shown in Fig. 2, and the different power levels can be seen in Fig. 3.

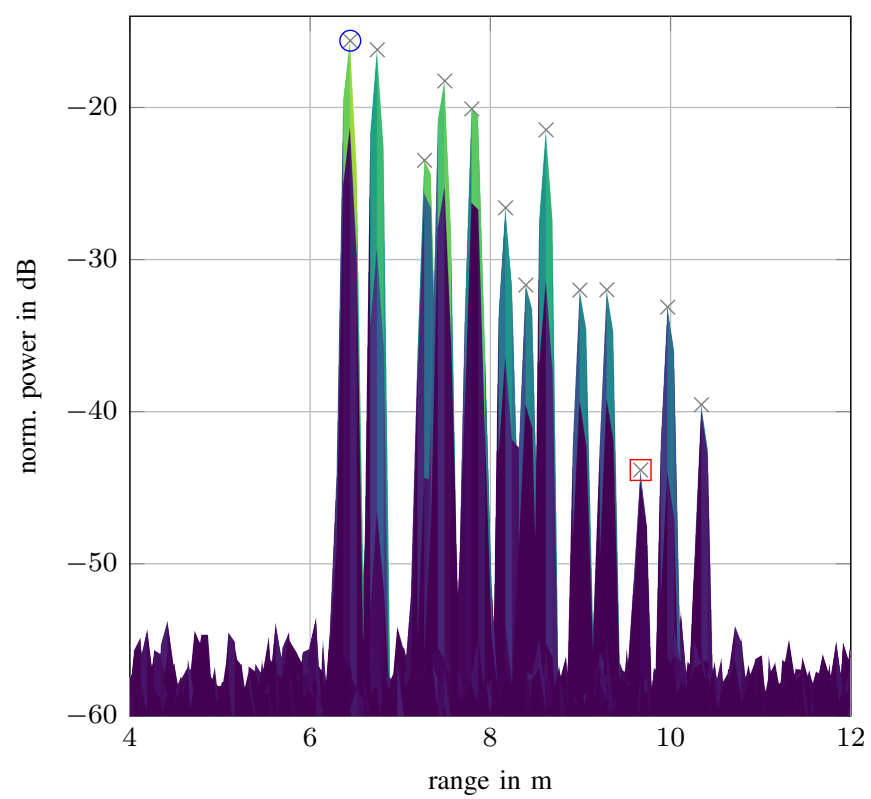

Fig. 3. Simulated scenario consisting of close targets in range and velocity. From the detected targets $(\times)$ the strongest $(\bigcirc)$ and the weakest $(\square)$ are selected for further analysis.

\section{A. Influence on Detection Rate}

As stated in the introduction and already shown in Fig. 2, it makes an important difference if complete frequency chirps or random samples are removed to gain an equal reduction of samples [6]. The influence on the detection rate of the chosen concept in combination with the percentage of samples present is shown in Fig. 4 (a). As for each single simulation the noise realisation and the pattern of removed samples is different, a weak target can be visible or not. Therefore, for every level of existing data several iterations are performed and the detection rate considers all expected targets averaged over all iterations with the same parameters.

It is noticeable that the detection rate of all targets drops the more samples are removed if no reconstruction of the missing data is applied. This effect is more severe for the removal of complete chirps (…...) than for random samples (---). This also holds for the detection rate of the reconstructed signal. If the weakest target has at least an $S N R$ of $15 \mathrm{~dB}$ as in the assumed scenario, a data rate reduction up to $60 \%$ is possible with a detection rate above $99.9 \%$.

TABLE I

SPECIFICATIONS OF RADAR PARAMETERS

\begin{tabular}{lr} 
Parameter & Value \\
\hline carrier frequency $f_{\mathrm{c}}$ & $76.5 \mathrm{GHz}$ \\
bandwidth $B$ & $2 \mathrm{GHz}$ \\
chirp duration $T_{\mathrm{c}}$ & $70 \mu \mathrm{s}$ \\
chirp repetition time $T_{\mathrm{r}}$ & $80 \mu \mathrm{s}$ \\
sampling frequency $f_{\mathrm{s}}$ & $25 \mathrm{MHz}$ \\
number of chirps $L$ & 256
\end{tabular}




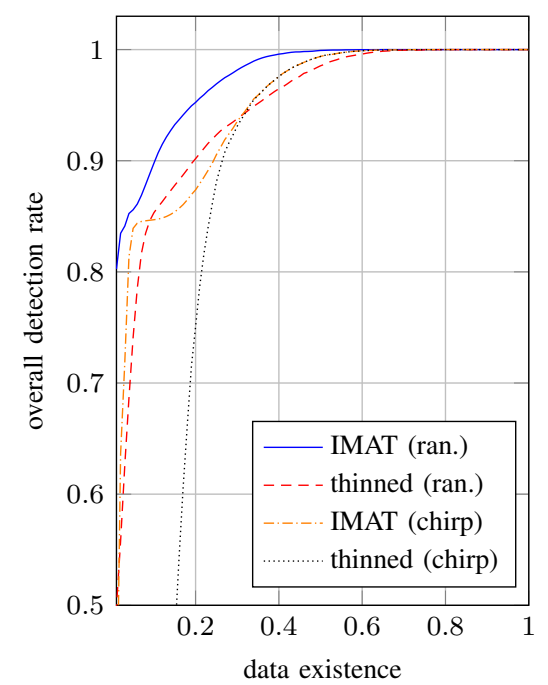

(a) Detection rate of all targets shown in Fig. 3.

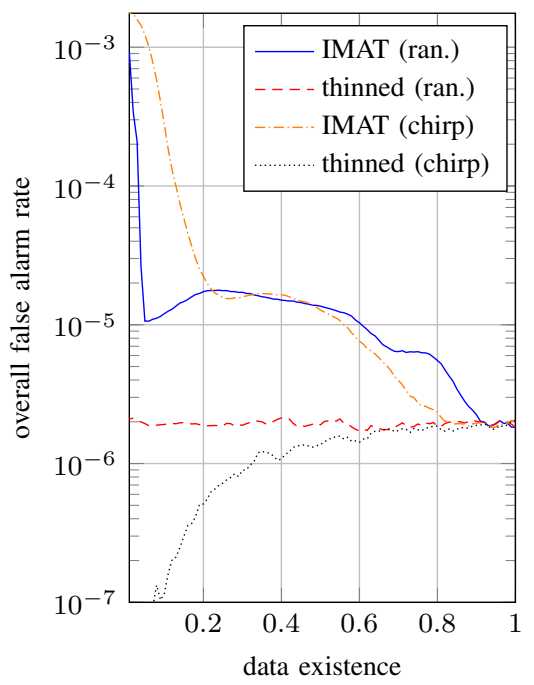

(b) False alarm rate after $O S-C F A R$.

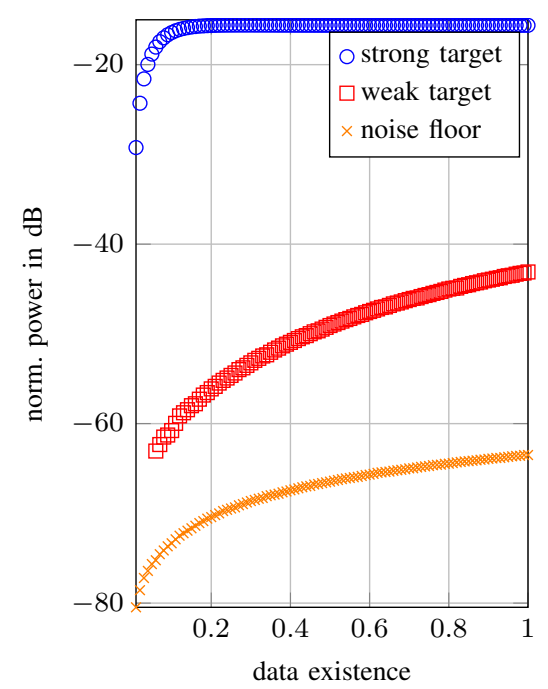

(c) Reconstructed power and noise floor.

Fig. 4. Simulation of the influence of missing samples on the detection rate in (a), the false alarm rate in (b), and on the reconstructed power and noise in (c). If only samples are removed the label 'thinned' is used for the removal of random ('ran.') samples or complete chirps ('chirp'). The application of the reconstruction algorithm is labelled with 'IMAT'. In (c) random samples are removed and reconstructed to estimate the power and noise levels.

\section{B. Influence on False Alarm Rate}

Every detected target after application of the OS-CFAR and the peak search, which was not simulated, is classified as a false alarm. The false alarm rate is shown in Fig. 4 (b). It can be seen that the false alarm rate of the randomly thinned signal (---) is nearly constant. The reason is that the more samples are removed the noise floor is more dominated by the artefact level induced by the missing samples. As there are not many large spikes in the spectrum, false alarms are low. Due to the fact that the targets are also loosing power due to less samples, the reconstruction algorithm cannot select the correct targets with certainty. Hence, some random noise peaks are treated as targets leading to an increased false alarm rate (-).

As can be noticed in Fig. 2 (a) the removal of whole chirps leads to rising ridges in the spectrum. As those ridges should be ignored for target detection, the OS-CFAR searches for targets in range cells only. If merely a few chirps are present for evaluation, the ridge is very dominant, and no target is found at all. This can be seen in the sudden drop of the detection rate and the false alarm rate (..........).

\section{Influence on Initial SNR}

The simulated scenario consists of targets with different power levels, cf. Fig. 3. For further analysis the strongest $(\bigcirc)$ and the weakest $(\square)$ target are of interest. The average noise power and the power of the reconstructed targets are shown in Fig. 4 (c). Removed samples are reconstructed only with the dominant signal parts, i.e. with information of the present targets and not with noise. This is the reason why the noise level is decreasing if more samples are neglected.

If a target is very dominant, e.g., it has a high $S N R$, it is also dominant in the thinned signal and can be reconstructed easily. Hence, the reconstructed peak power of the strong target $(O)$ is constant for a long period. Weak targets are harder to detect in the thinned signal and are only detected in the last iterations of the algorithm. Hence, the reconstruction is possible but the original peak power of the full signal cannot be reached. If too many samples are missing, the weak target is not detected anymore as can be seen by the sudden end of the reconstructed power ( $\square$ ).

The detection rate of weak targets can be enhanced if the threshold of the algorithm is lowered, but then the false alarm rate rises. Therefore, a trade-off has to be made between a reasonable detection and false alarm rate. This also holds for the parametrisation of target detection algorithms.

\section{Measurement Evaluation}

To verify the simulation results a measurement with an experimental radar sensor is performed. The parameters of the chirp-sequence modulation are the same as for the simulation and listed in Tab. I. The radar sensor uses two $25 \mathrm{dBi}$-gain horn antennae with a $3 \mathrm{~dB}$ beamwidth of $7^{\circ}$ each. Hence, the illuminated area is comparable small and therefore, a walking

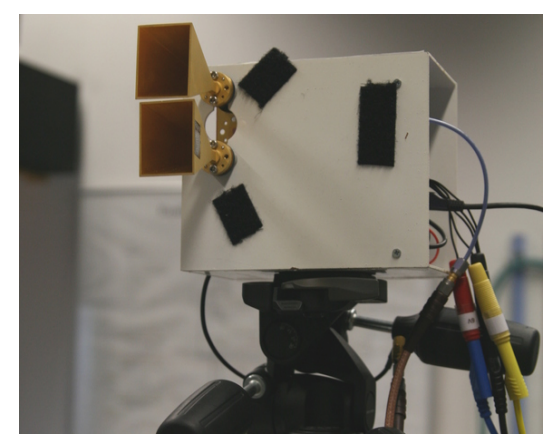

Fig. 5. Photo of the experimental radar sensor with the two $25 \mathrm{dBi}$-gain horn antennae. 


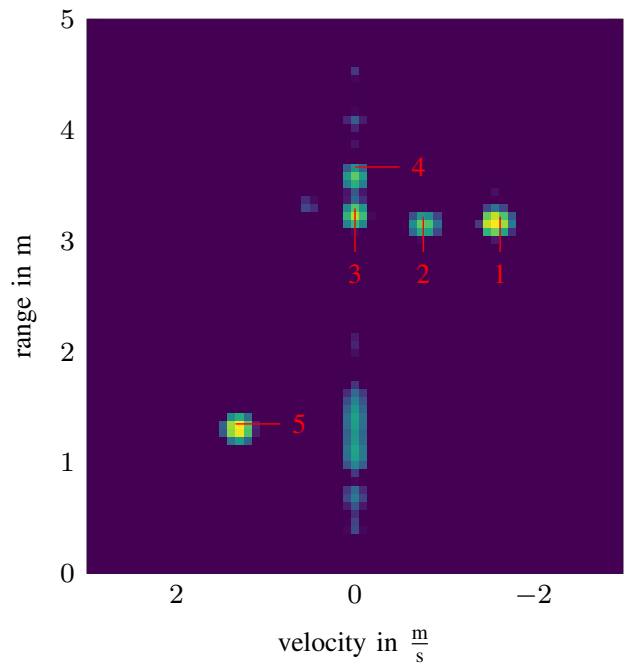

(a) Range-Doppler evaluation using the full signal.

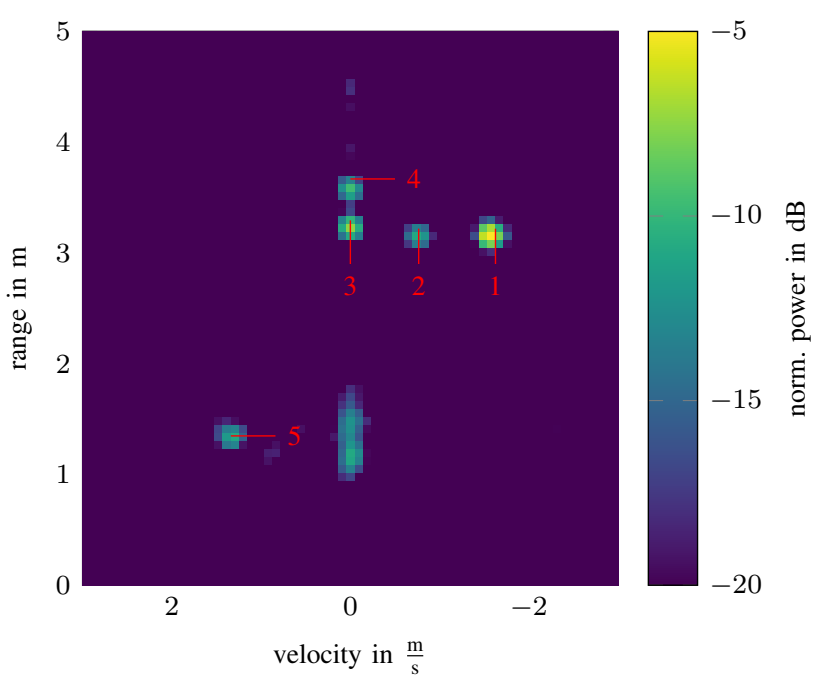

(b) Range-Doppler evaluation with only $40 \%$ of the samples left. Missing samples reconstructed with IMAT algorithm.

Fig. 6. Measurement of a walking pedestrian with two scattering centres (1 and 2). Evaluation of the full signal in (a) and with $60 \%$ removed samples in (b).

TABLE II

COMPARISON OF TARGET POWER LEVEls OF FIG. 6

\begin{tabular}{crr} 
Target & Power Original & Power Reconstructed \\
\hline 1 & $-4.2 \mathrm{~dB}$ & $-4.6 \mathrm{~dB}$ \\
2 & $-8.6 \mathrm{~dB}$ & $-10.9 \mathrm{~dB}$ \\
3 & $-6.7 \mathrm{~dB}$ & $-7.5 \mathrm{~dB}$ \\
4 & $-8.7 \mathrm{~dB}$ & $-9.6 \mathrm{~dB}$ \\
5 & $-5.1 \mathrm{~dB}$ & $-11.0 \mathrm{~dB}$
\end{tabular}

pedestrian is considered as target. A photo of the sensor is shown in Fig. 5.

The range-Doppler evaluation of the walking pedestrian is shown in Fig. 6 (a). The two prominent scattering centres 1 and 2 are detected, while 3 and 4 are stationary targets in the laboratory. Unfortunately, an internal interferer is always present, which is target 5. To suppress the $D C$ peak a digital high-pass filter is applied. Otherwise the reconstruction algorithm would amplify the $D C$ component too much.

With a data reduction down to only $40 \%$ of the samples, in most cases every target should be detectable regarding Fig. 4 (a). For that reason $60 \%$ of the samples are removed and the IMAT algorithm is used for reconstruction. The processed range-Doppler evaluation is shown in Fig. 6 (b). Tab. II compares the peak power of the full signal to the reconstructed one. All target peaks can be successfully recovered after the application of the IMAT algorithm. As the parametrisation of the reconstruction algorithm is optimised, no noise clutter is amplified.

\section{CONCLUSION}

In order to reduce the data rate of a chirp-sequence radar sensor random samples are removed and later on reconstructed using a compressed sensing approach. This lowers the number of required analog-to-digital converters. It is shown that the removal of random samples has a significant advantage over the removal of complete chirps. Simulations show the data rate reduction that is achieved without loosing the detection of weak targets. If a target has an SNR of roughly $15 \mathrm{~dB}$ a reduction of $60 \%$ of samples offers a detection rate of $99.9 \%$. The results are verified with a measurement of a walking pedestrian.

\section{REFERENCES}

[1] J. Dickmann, J. Klappstein, M. Hahn, N. Appenrodt, H.-L. Bloecher, K. Werber, and A. Sailer, "'Automotive Radar the Key Technology for Autonomous Driving: From Detection and Ranging to Environmental Understanding"," in IEEE Radar Conference, May 2016, pp. 1-6.

[2] H.-L. Blöecher, M. Andres, C. Fischer, A. Sailer, M. Goppelt, and J. Dickmann, "Impact of system parameter selection on radar sensor performance in automotive applications," Advances in Radio Science, vol. 10, pp. 33-37, 2012.

[3] D. L. Donoho, "Compressed Sensing," Transactions on Information Theory, vol. 52, no. 4, pp. 1289-1306, Apr. 2006.

[4] E. J. Candès, J. K. Romberg, and T. Tao, "Stable Signal Recovery from Incomplete and Inaccurate Measurements," Communications on Pure and Applied Mathematics, vol. 59, no. 8, pp. 1207-1223, Mar. 2006. [Online]. Available: http://dx.doi.org/10.1002/cpa.20124

[5] S. Lutz, D. Ellenrieder, T. Walter, and R. Weigel, "On fast chirp Modulations and Compressed Sensing for Automotive Radar Applications," in 15th International Radar Symposium (IRS), Jun. 2014, pp. 1-6.

[6] G. Lellouch, P. Tran, R. Pribic, and P. van Genderen, "Ofdm waveforms for frequency agility and opportunities for doppler processing in radar," in IEEE Radar Conference, May 2008, pp. 1-6.

[7] V. Winkler, "Range Doppler Detection for automotive FMCW Radars," in Proceedings of the 4th European Radar Conference (EuRAD), Oct. 2007, pp. 166-169.

[8] F. Marvasti, A. Amini, F. Haddadi, M. Soltanolkotabi, B. H. Khalaj, A. Aldroubi, S. Holm, S. Sanei, and J. A. Chambers, "A Unified Approach to Sparse Signal Processing," CoRR, vol. abs/0902.1853, 2009. [Online]. Available: http://arxiv.org/abs/0902.1853

[9] H. Rohling, "Radar CFAR Thresholding in Clutter and Multiple Target Situations," IEEE Transactions on Aerospace and Electronic Systems, vol. AES-19, no. 4, pp. 608-621, Jul. 1983.

[10] M. Bühren and B. Yang, "Automotive Radar Target List Simulation based on Reflection Center Representation of Objects," in Workshop on Intelligent Transportation (WIT), Hamburg, Germany, Mar. 2006. 